\title{
Review of: "Knowledge, Awareness, and Practices (KAP) towards COVID-19 among the marine fishers of Maharashtra State of India: An online cross-sectional Analysis"
}

\section{P Ravi Shankar}

Potential competing interests: The author(s) declared that no potential competing interests exist.

Thank you for the invitation to review this manuscript. The study is important and as the authors examine knowledge, attitude, and practice among a vulnerable and marginalized community. The study is mostly well-written and presented.

There is some information that is missing. Where exactly was the study carried out? What was the population being studied? Does the sample reflect the overall population? Who were the persons chosen? Was this both the fishermen and their family members? What were the inclusion and the exclusion criteria of respondents?

Why was consent not obtained? Or was the consent implied? Why did the authors not obtain approval from an ethical board? Though there was minimal risk to the respondents these issues may be important as fishers may be regarded as a vulnerable population as mentioned by the authors.

Another point is that with COVID-19 information rapidly changes and some of the information mentioned in the manuscript has now changed. I am aware that this is mainly because of the long-time interval required for multiple reviews and publication of research manuscripts. Do the authors want to make any specific recommendations for policy and practice? What about further research which may be required? Some language corrections may be required in the Abstract. 